\title{
Detection and Classification of Leukemia using MPFCM Segmentation and Random Forest with Boosting Techniques
}

\author{
T.C.Kalaiselvi
}

Assistant Professor, Department of ECE , Kongu Engineering College,Perundurai, Tamil Nadu, India .kalaiselvi@kongu.ac.in

\section{ABSTRACT:}

Identification of blood disorders is through visual inspection of microscopic blood cell images. From the identification of blood disorders lead to classification of certain diseases related to blood. We propose an automatic segmentation method for segmenting White blood cell images. Firstly, modified possibilistic fuzzy c-means algorithm is proposed to detect the contours in the image. The GLCM features are extracted and features are selected by MRMR. Adaptive boosting and LS Boosting has been utilized to classify blast cells from normal lymphocyte cells. Comparison performance of classification accuracy was carried out. The effectiveness of the classification system is tested with the total of 80 samples collected. The evaluated results demonstrate that our method outperformed the existing systems with an accuracy of $88 \%$.

\section{Keywords-MPFCM segmentation, ADA Boosting LS Boosting, GLCM features, MRMR Feature selection}

\section{Introduction}

The assessment of the white blood cells in the bone marrow of patients is very informative in clinical practice. Segmentation is the process of partitioning a digital image into multiple segments based on pixels[7]. Segmentation is a critical and essential component of image analysis system[8]. The result of image segmentation is a collection of segments which combine to form the entire image. Various techniques have been proposed for segmenting an image in a better way. From the segmentation result, geometrical features such as area, perimeter etc [1] were detected for the final detection of immature cells. Three different classification techniques such as Tree Bagger, LS Boosting and ADA boosting were employed for classification[4] [5] [6], in order to classify the lymphocyte (WBC) as healthy and leukemic.

\section{Proposed Methodology}

\subsection{MPFCM Clustering Segmentation}

Image segmentation is the process of partitioning

a digital image into multiple segments. Image segmentation is typically used to locate objects and boundaries in images [1].MPFCM is a good clustering algorithm [2] [3] to perform classification tests because it possesses capabilities to give more importance to typicalities or membership values. In order to avoid the constraint corresponding to the sum of all typicality values of all data to a cluster must be equal to one cause problems particularly for a big data set. It produces memberships and possibilities simultaneously, with the usual point prototypes or cluster centers for each cluster. The objective function is defined by

$$
\begin{aligned}
J_{p f c m}=\sum_{i=c}^{c} \sum_{k=1}^{n}\left(\mathrm{a} \mu_{i k}^{m}+\mathrm{b} t_{i k}^{\eta}\right) \times\left\|z_{k}-v_{i}\right\|^{2} \\
\\
\quad+\sum_{i=1}^{c} \mathrm{Yi}^{\sum_{k=1}^{n}\left(1-t_{i k}\right)^{\eta}}
\end{aligned}
$$

Subject to the constraints $\sum_{i=1}^{c} \mu_{i k}=1$ for all $\mathrm{k}$ and

$0 \leq \mu_{i k}, \leq 1$. Here $\mathrm{a}>0, \mathrm{~b}>0, \mathrm{~m}>0$, and $\eta>0$. $U$ is the partition matrix. $\mathrm{T}$ is the typicality matrix. $\mathrm{V}$ is a vector of cluster centers, $\mathrm{X}$ is a set of all data points, $\mathrm{z}$ represents a data point, $\mathrm{n}$ is the number of data points and $\mathrm{c}$ is the number of cluster centers which are described by s coordinates.

\subsubsection{Algorithm}

Step 1: Initialize prototype

$$
\begin{aligned}
v i= & \sum_{k=1}^{N}\left(a \mu_{i k}^{m}+b t_{i k}^{\eta}\right) Z_{k} / \sum_{k=1}^{N}\left(a \mu_{i k}^{m}+b t_{i k}^{\eta}\right) \\
& 1 \leq \mathrm{i} \leq \mathrm{C}
\end{aligned}
$$

Step 2: For each cluster compute penalty parameter $\gamma_{i}$

$\gamma_{i=\mathrm{K}} \sum_{k=1}^{n} \mu_{i k}^{m}\left\|\mathrm{Z}-v_{i}\right\|^{2}$

Step 3: For each prototype calculate the distance

$$
\begin{aligned}
D_{i k A}^{2}= & \left(z_{k}-v_{i}\right)^{T} A_{i}\left(z_{k}-v_{i}\right), 1 \leq \mathrm{i} \leq \mathrm{C}, \\
& 1 \leq k \leq N
\end{aligned}
$$

Step 4: Calculate membership and typicality values

$$
\begin{aligned}
\mu_{i k}= & \left(\sum_{j=1}^{c}\left(\frac{D_{i k A_{i}}}{D_{j k A_{i}}}\right)^{2 /(m-1)}\right)^{-1}, 1 \leq \mathrm{i} \leq \mathrm{C}, \\
& 1 \leq k \leq N
\end{aligned}
$$


$t_{i k}=\frac{1}{1+\left(\frac{b}{r_{i}} D_{i: A_{i}}^{2}\right)^{1 /\left(V^{-1)}\right.}}, 1 \leq \mathrm{i} \leq \mathrm{C}, 1 \leq k \leq N$

(6)

Step 5: Update the values of prototypes.

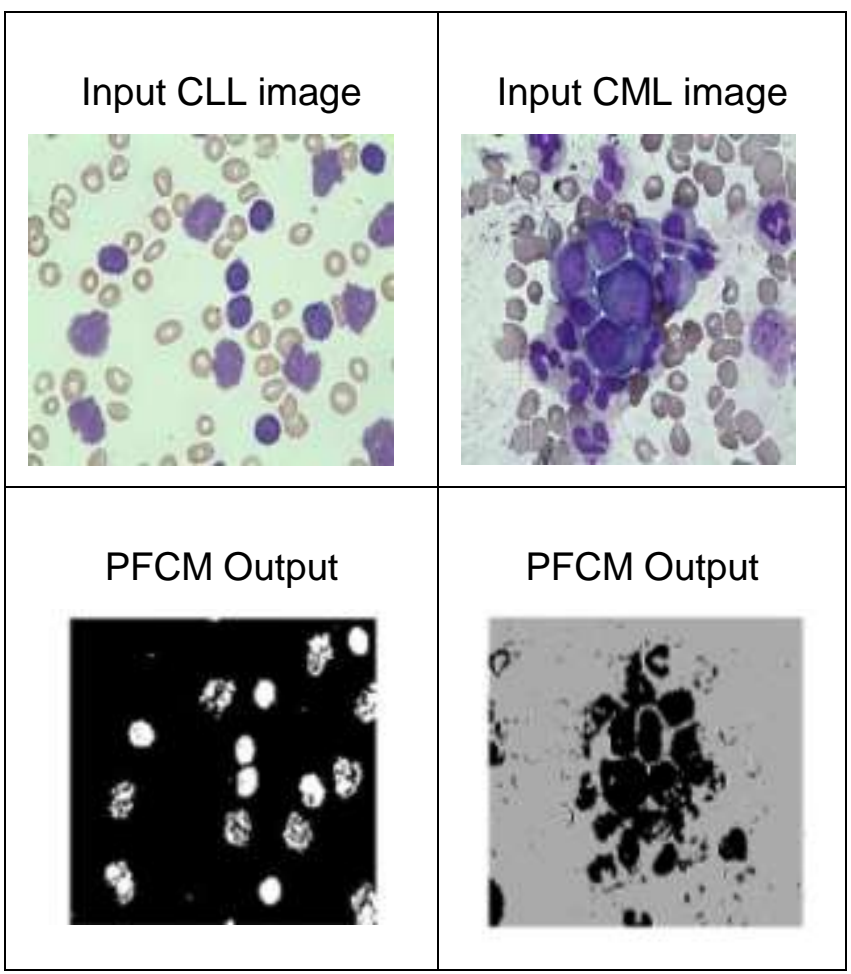

Fig.1 Input and Segmented Output of CLL \& CML Table.1 Evaluation Parameters of PFCM

\subsection{Feature Extraction}

\begin{tabular}{|c|c|}
\hline EVALUATION & PFCM \\
PARAMETERS & 0.9922 \\
\hline Rand Index & 0.9866 \\
\hline Jaccard Index & 2.0000 \\
\hline Dice Coefficient & \\
\hline
\end{tabular}

Feature extraction is a special form of dimensionality reduction [1]. When the input data to an algorithm is too large to be processed, then the input data will be transformed into a reduced representation set of features. Textural features based on the gray level co-occurence matrix (GLCM) are extracted from each image that are used to distinguish between normal and abnormal cancer cells. Co-occurrence matrices are calculated for four directions: $0^{\circ}, 45^{\circ}, 90^{\circ}$ and $135^{\circ}$ degrees.

GLCM has following features: Autocorrelation, Contrast, Correlation, Cluster Prominence, Cluster Shade, Dissimilarity, Energy, Entropy, Homogeneity, Maximum probability, Sum of squares, Sum average, Sum variance, Sum entropy, Difference variance, Difference entropy, Information measure of correlation, information measure of correlation, Inverse difference normalized are listed in table.2 
Table.2 Feature Extraction Result

\begin{tabular}{|l|l|l|l|}
\hline Features & Normal & CLL & CML \\
\hline Contrast & 1.1912 & 2.8458 & 1.6078 \\
\hline correlation & 0.9994 & 0.9986 & 0.9987 \\
\hline $\begin{array}{l}\text { cluster } \\
\text { prominence }\end{array}$ & 1610 & 2132 & 1171 \\
\hline Cluster shade & -2510 & -3092 & -1976 \\
\hline dissimilarity & 0.2549 & 0.4516 & 0.4005 \\
\hline energy & 0.3964 & 0.423 & 0.3414 \\
\hline entropy & -0.3278 & -0.346 & -0.2818 \\
\hline homogeneity & 0.9428 & 0.9304 & 0.8996 \\
\hline Max probability & 0.478 & 0.5347 & 0.5126 \\
\hline Sum of squares & 28.2088 & 38.0687 & 21.3773 \\
\hline Sum of average & 1.1912 & 2.8458 & 1.6078 \\
\hline Sum of variance & 0.9994 & 0.9986 & 0.9987 \\
\hline Sum entropy & 1610 & 2132 & 1171 \\
\hline Diff variance & -2510 & -3092 & -1976 \\
\hline Diff entropy & 0.2549 & 0.4516 & 0.4005 \\
\hline $\begin{array}{l}\text { Info measure of } \\
\text { correlation 1 }\end{array}$ & 0.3964 & 0.423 & 0.3414 \\
\hline $\begin{array}{l}\text { Info measure of } \\
\text { correlation 2 }\end{array}$ & -0.3278 & -0.346 & -0.2818 \\
\hline $\begin{array}{l}\text { Inverse diff } \\
\text { normalized }\end{array}$ & 0.9428 & 0.9304 & 0.8996 \\
\hline $\begin{array}{l}\text { Inverse diff } \\
\text { moment } \\
\text { normalized }\end{array}$ & 0.478 & 0.5347 & 0.5126 \\
\hline autocorrelation & 28.2088 & 38.0687 & 21.3773 \\
\hline
\end{tabular}

\subsection{Feature Selection}

Feature selection is the process of selecting a subset of relevant features for use in model construction. Features are selected based on MRMR and tabulated in table.3.

\subsubsection{Minimum-Redundancy and Maximum-Relevance (MRMR)}

Feature-selection method that can use either mutual information, correlation, distance/similarity scores to select features [10]. The relevance of a feature set $S$ for the class $c$ is defined by the average value of all mutual information values between the individual feature $x_{i}$ and the class $c$ as follows

$$
\mathrm{D}=\frac{1}{|\mathrm{~S}|} \sum_{\mathrm{x}_{\mathrm{i}} \in \mathrm{S}} \mathrm{I}\left(\mathrm{x}_{\mathrm{i}} ; \mathrm{c}\right)
$$

The redundancy of all features in the set $S$ is the average value of all mutual information values between the feature $x_{i}$ and the feature $x_{j}$ :

$$
\mathrm{R}=\frac{1}{|\mathrm{~S}|^{2}} \sum_{\mathrm{x}_{\mathrm{i}}, \mathrm{x}_{\mathrm{j}} \in \mathrm{S}} \mathrm{I}\left(\mathrm{x}_{\mathrm{i}} ; \mathrm{x}_{\mathrm{j}}\right)
$$

The MRMR criterion is a combination of two measures given above and is defined as follows

$\operatorname{mRMR}=\max _{\mathrm{S}}\left[\frac{1}{|\mathrm{~S}|} \sum_{\mathrm{x}_{\mathrm{i}} \in \mathrm{S}} \mathrm{I}\left(\mathrm{x}_{\mathrm{i}} ; \mathrm{c}\right)-\frac{1}{|\mathrm{~S}|^{2}} \sum_{\mathrm{x}_{\mathrm{i}}, \mathrm{x}_{\mathrm{j}} \in \mathrm{S}} \mathrm{I}\left(\mathrm{x}_{\mathrm{i}} ; \mathrm{x}_{\mathrm{j}}\right)\right](9)$

Suppose that there are $n$ full-set features. Let $f_{i}$ be the set membership indicator function for feature $x_{i}$, so that $f_{i}=1$ indicates presence and $f_{i}=0$ indicates absence of the feature $x_{i}$ in the globally optimal feature set. Let $c_{i}=I\left(x_{i} ; c\right)$ and $a_{i j}=I\left(x_{i} ; x_{j}\right)$.

The above may then be written as an optimization problem

$\left.\operatorname{MRMR}=\max _{\mathrm{f} \in\{0,1}\right\}^{\mathrm{n}}\left[\frac{\sum_{\mathrm{i}=1}^{\mathrm{n}} \mathrm{c}_{\mathrm{i}} \mathrm{f}_{\mathrm{i}}}{\sum_{\mathrm{i}=1}^{\mathrm{n}} \mathrm{f}_{\mathrm{i}}}-\frac{\sum_{\mathrm{i}, \mathrm{j}=1}^{\mathrm{n}} \mathrm{a}_{\mathrm{ij}} \mathrm{f}_{\mathrm{i}} \mathrm{f}_{\mathrm{j}}}{\left(\sum_{\mathrm{i}=1}^{\mathrm{n}} \mathrm{f}_{\mathrm{i}}\right)^{2}}\right]$ 
Table.3 Feature Selection Result

\begin{tabular}{|c|c|c|c|}
\hline Features & Normal & CLL & CML \\
\hline Contrast & 1.1912 & 2.8458 & 1.6078 \\
\hline $\begin{array}{c}\text { Cluster } \\
\text { prominence }\end{array}$ & 1610 & 2132 & 1171 \\
\hline Cluster shade & -2510 & -3092 & -1976 \\
\hline Sum of squares & 28.2088 & 38.0687 & 21.3773 \\
\hline Sum of average & 6.8499 & 8.3153 & 5.2302 \\
\hline Sum of variance & 70.1377 & 101.1808 & 47.8642 \\
\hline Sum entropy & 0.8094 & 0.7115 & 1.1627 \\
\hline Diff variance & 1.1912 & 2.8458 & 1.6078 \\
\hline Diff entropy & 0.3995 & 0.424 & 0.676 \\
\hline Autocorrelation & 27.7618 & 36.7772 & 20.6703 \\
\hline
\end{tabular}

\subsection{Classification}

Classification is the task of assigning to the unknown test vector, a label from one of the known classes. Classification methods aimed to find mathematical models to recognize the membership of each object to its proper class on the basis of a set of measurements [7]. Once a classification model has been obtained [8], the membership of unknown objects to one of the defined classes can be predicted.

\subsubsection{LS Boosting}

- It fits regression ensembles

- At every step, the ensemble fits a new learner to the difference between the observed response and the aggregated prediction of all learners grown previously.

- The ensemble fits every new learner to $Y_{n}-\eta f\left(x_{n}\right)$, Where, $Y_{n}$ - observed response, $f\left(x_{n}\right)$ - aggregated prediction from all grown weak learners,

$\eta$ - learning rate $(0-1)$

\subsubsection{ADA Boosting}

ADA Boost is a machine learning algorithm [4] that boosts the performance of other learning algorithms, known as weak learners, by weighting and combining them. The basic idea is that multiple weak learners can be combined to generate a more accurate ensemble, known as a strong learner. Various versions of the ADA Boost algorithm [6] have proven to be very competitive in terms of prediction accuracy in a variety of applications.

\section{Performance Measures}

The final results of the ensemble classifiers were analyzed and its performance evaluation is done based on the results obtained from the confusion matrix. Also the results are compared based on the three parameters namely accuracy, specificity and sensitivity.

\subsection{Confusion Matrix}

A confusion matrix is a specific table layout that allows visualization of the performance of an algorithm, typically a supervised learning .A table of confusion, is a table with two rows and two columns that reports the number of false positives, false negatives, true positives, and true negatives. The table of confusion includes True Positive(Cancer identified as Cancer), True Negative(Cancer identified as Non Cancer), False Positive(Non Cancer identified as Non Cancer) ,False Negative (Non Cancer identified as Cancer)

\subsection{Performance Parameters}

The parameters are namely

(i) Accuracy - statistical measure of how well a binary classification test correctly identifies or excludes a condition.

No of true positives + No of true negatives

\footnotetext{
Accuracy $=\overline{\text { True positives }+ \text { false positives }+ \text { false negatives }}$ + true negatives
} 
(i.e) Accuracy $=(T P+T N) /(T P+F P+T N+F N)$

where TP-True positive, TN-true negative,

TN-true negative, FN-false negative

(ii) Sensitivity -Measures the proportion of actual positives which are correctly identified as such and it is complementary to the false negative rate.

$$
\begin{aligned}
& \text { Sensitivity }=\quad \frac{\text { Number of true positives }}{\text { (Number of true positives }+ \text { Number of false }} \\
& \text { negatives) }
\end{aligned}
$$

(iii) Specificity - Measures tne proportıon or negatıves wnıch are correctly identified as such and is complementary to the false positive rate. Mathematically, this can also be written as

Specificity $=$

(i.e.) Specificity $=\mathrm{TN} /(\mathrm{TN}+\mathrm{TP})$

Number of true negatives

Number of true negatives + Number

\section{Results} of false positives)

\subsection{LS Boosting}

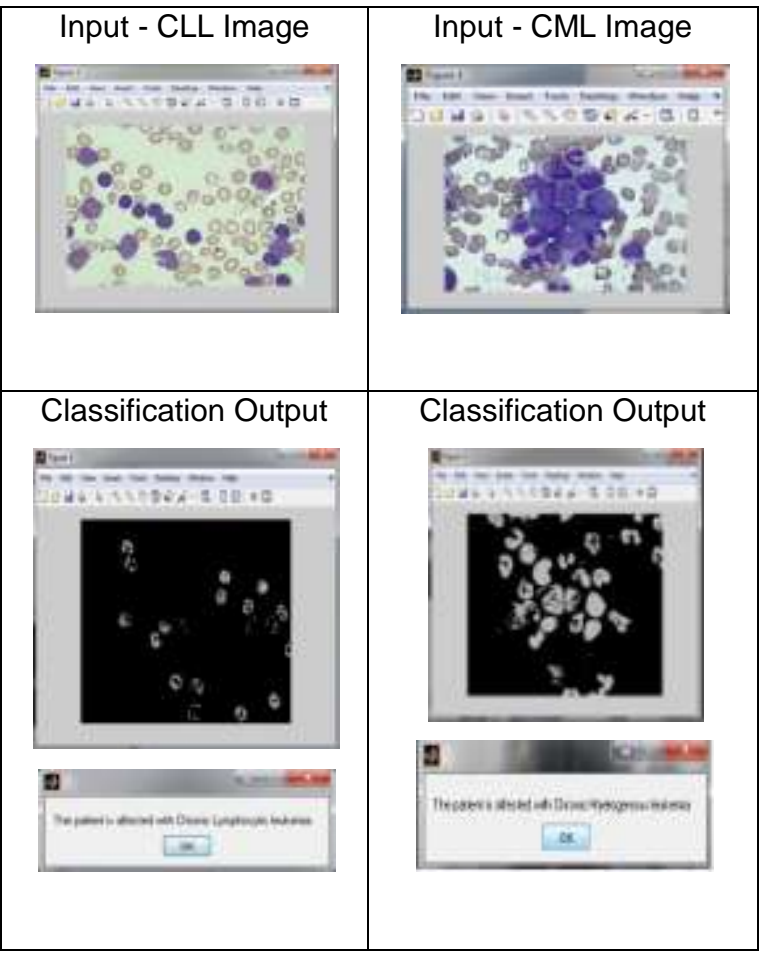

Fig.2 Classification Result for LS Boosting

\subsection{Adaptive Boosting}




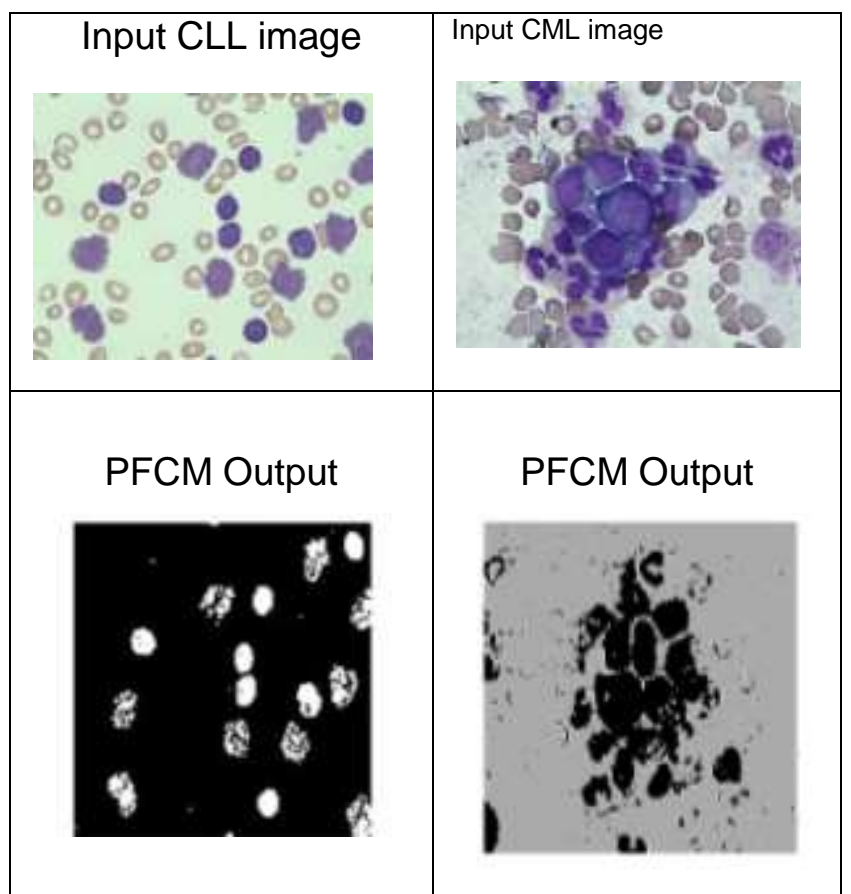

Fig.3 Classification Result for Adaptive boosting

\subsection{Performance Comparison}

The accuracy, specificity and sensitivity of adaptive and LS boosting is tabulated in table.4 comparison is done as shown in fig. 4 where LS boosting has high performance

Table.4 Performance Comparison of ADA and LS Boosting

\begin{tabular}{|c|c|c|}
\hline Algorithm/Parameters & $\begin{array}{c}\text { Adaptive } \\
\text { Boosting }\end{array}$ & $\begin{array}{c}\text { LS } \\
\text { Boosting }\end{array}$ \\
\hline Accuracy & $72.22 \%$ & $88.88 \%$ \\
\hline Specificity & 0.5833 & 0.8333 \\
\hline Sensitivity & 0.7917 & 0.9167 \\
& & \\
\hline
\end{tabular}

\section{Performance Comparison}

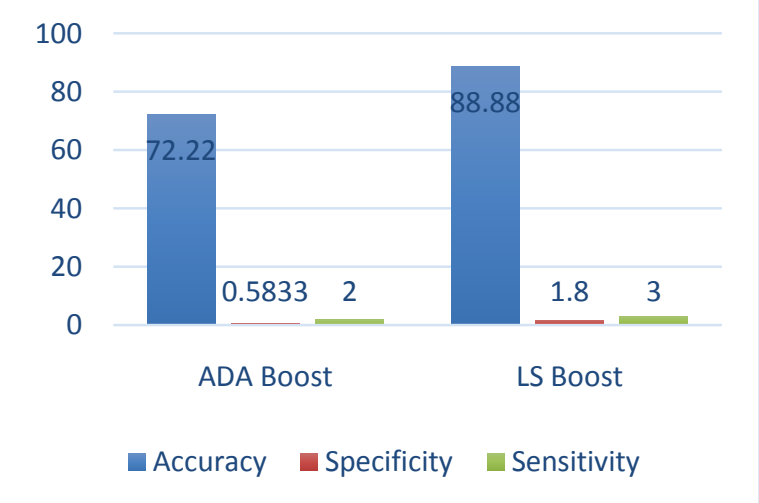

Fig.4 Performance Comparison of Classification algorithm 


\section{Conclusion}

Thus the modified possibilistic fuzzy c-means algorithm approach was evaluated and tested for various blood cells. This algorithm gives the segmentation result of white blood cells. This algorithm begins with detecting the cells in the region. By using those regions, white blood cells alone segmented. Then classification algorithms such as adaptive boosting and LS boosting were implemented and performance is measured.

\section{References}

[1] Athira Krishnan, Sreekumar K, A Survey On Image Segmentation And Feature Extraction Methods For Acute Myelogenous Leukemia Detection in Blood Microscopic Images, International Journal Of Computer Science And Information Technologies, Vol. 5 (6) , 7877-7879,2014

[2] Arindam chaudhuri, intuitionistic fuzzy possibilistic c means clustering algorithms, advances in fuzzy systems, article id 238237, volume 2015

[3] Aruna bhat,possibility fuzzy c-means clustering for expression invariant face recognition, international journal on cybernetics \& informatics vol. 3, no. 2, april 2014

[4] Claudia Henry Richard Nock, Frank Nielsen,Real Boosting a la Carte with an Application to Boosting Oblique Decision Trees, IJCAI-07

[5] Esteban Alfaro, Noelia García, MatíasGámez, and David Elizondo( 2008). Bankruptcy forecasting: 'An empirical comparison of AdaBoost and neural networks'. DecisionSupport Systems, 45(1):110_122.

[6] Gall, J., Yao, A., Razavi, N., Van Gool, L., and Lempitsky,V. (2011)' Hough forests for object detection, tracking, and action recognition'. InPattern Analysis andMachine Intelligence.IEEE.

[7] Genuer, R., Poggi J.M., Tuleau-Malot C (2010)‘Variable Selection Using Random Forests', Pattern Recognition Letters, vol. 31, pp. 2225-2236.

[8] Harun.N.H, A. S. Abdul Nasir, M. Y. Mashor, R. Hassan Unsupervised Segmentation Technique For Acute Leukemia Cells Using Clustering Algorithms International Journal Of Computer, Control, Quantum And Information Engineering Vol:9, No:1, 2015

[9] Hongwei hu , bo ma , yuwei wu , weizhang ma and kai xie,kernel regression based online boosting tracking ${ }^{\star}$, journal of information science and engineering 31, 267-282 (2015)

[10] Hudson F. Golino1, Cristiano Mauro Assis Gomes2,Visualizing Random Forest's Prediction

Results, Published Online December 2014 in SciRes. http://www.scirp.org/journal/psych

[11] Kalaiselvi Chinnathambi, Asokan Ramasamy, Premkumar Rajendran, An Effective Clustering Technique For Wbc Image Segmentation And Its Classification, International Journal Of Digital Signal And Image Processing Vol. 2, No. 1(March 2014)

[12] Kulkarni V Y, Sinha P K, Petare M (2012), 'Analyzing Random Forest Classifier using Different Split Measures', Proceedings of International Conference on Soft Computing and Problem Solving, Jaipur, India. , Springer AISC series.

[13] Nazeeh Ghatasheh, Business Analytics using Random Forest Trees for Credit Risk Prediction: A Comparison Study, International Journal of Advanced Science and Technology Vol.72 (2014), pp.19-30

[14] Nicolas De Jay, Simon Papillon-Cavanagh , Catharina Olsen2,an R package for parallelized mRMR ensemble feature selection, August 19, 2014

[15] Vrushali Y Kulkarni, Pradeep K Sinha ,Effective Learning and Classification using Random Forest Algorithm, International Journal of Engineering and nnovative Technology (IJEIT) Volume 3, Issue 11, May 2014. 